\title{
A case of congenital malformations of the genital tract diagnosed in a 44-year-old woman: non-fusion of the mullerian duct
}

Received: 24 January 2006 / Accepted: 9 April 2006 / Published online: 24 August 2006

(C) Springer-Verlag Berlin / Heidelberg 2006

\begin{abstract}
We present a case of congenital malformations of the genital tract diagnosed in a 44-year-old woman. This case demonstrates that the congenital disorders of development are more common than previously reported and the correct diagnosis is frequently missed, leading to suboptimal management.
\end{abstract}

Keywords Abdominal pain · Mullerian abnormality · Fibroids

\section{Case report}

A 44-year-old nulliparous woman was referred with a history of lower abdominal pain for many years, which had been getting worse for 1 year. She had never had menarche. She was otherwise well, apart from having well-controlled asthma, and had no history of previous surgery. On examination, the external genitalia were normal. The cervix and upper third of the vagina were absent. A large mass extending beyond the umbilicus was noted abdominally.

Full blood count revealed a normal haemoglobin and normal white cell count. The sonograms revealed a grossly enlarged uterus measuring $13.3 \times 10.4 \times 17.6 \mathrm{~cm}$. Multiple fibroids were noted. Normal ovaries, gall bladder and kidneys were noted. She was admitted for laparotomy, abdominal hysterectomy and bilateral salpingo-oophorectomy.

F. Bano $\cdot$ N. Rafla

Department Of Obstetrics and Gynaecology,

Queen Elizabeth the Queen Mother Hospital,

CT9 4AN Margate, Kent, UK

F. Bano $(\bowtie)$

Department of Obstetrics and Gynaecology,

King's College Hospital,

Denmark Hill,

SE5 9RS, London, UK

e-mail: faridabano@hotmail.com

Tel.: +44-795-1696753

Fax: +44-184-3295915
Midline abdominal incision was performed. The procedure was difficult due to multiple adhesions between the bowel and uterus. Due to the absent cervix and large fibroids, the uterus was attached to the pelvis with a broad base, making access difficult particularly to the uterine vessels. There were multiple large fibroids amounting to 24-week size which were removed separately. The largest fibroid was noted to be arising from the two horns of the defective Mullerian fusion system. There were two small uteri, each with an ovary and tube attached to a large mass (Fig. 1a,b). An estradiol implant was inserted at the time of operation for relief of menopausal symptoms.

Postoperatively, the patient made a good recovery. She received prophylactic antibiotics and low molecular weight heparin. She was discharged home 4 days after her surgery. She was followed up in the clinic and reported to have complete resolution of pain and was asymptomatic.

The histopathology report showed uterine corpus together with both appendages weighing $1,123 \mathrm{~g}$. The uterus was replaced by a large intramural fibroid measuring $130 \times 110 \times 80 \mathrm{~mm}$. The endometrial cavity appeared obliterated. There was no evidence of necrosis within the fibroid mass. The left fallopian tube was unremarkable and measured $70 \mathrm{~mm}$ long and the attached ovary measured $40 \times 30 \mathrm{~mm}$. The right fallopian tube was unremarkable and measured $68 \mathrm{~mm}$ long and the right ovary measured $50 \times 20 \times 20 \mathrm{~mm}$. Two fibroid masses together weighed $397 \mathrm{~g}$. The larger fibroid mass measured $120 \times 90 \times 50 \mathrm{~mm}$. It confirmed benign leiomyomata. The ovaries contained corpora luteal cysts. No endometrial lining was seen.

\section{Discussion}

Congenital disorders of development are more common than previously reported and the correct diagnosis is frequently missed, leading to suboptimal management. Uterovaginal anomalies can be a challenging group of conditions. Maldevelopment of the Mullerian duct system may result in various urogenital anomalies, including didelphic or rudimentary uterus with non-canalisation 
Fig. 1 Two small uteri, each with an ovary and tube attached to a large mass
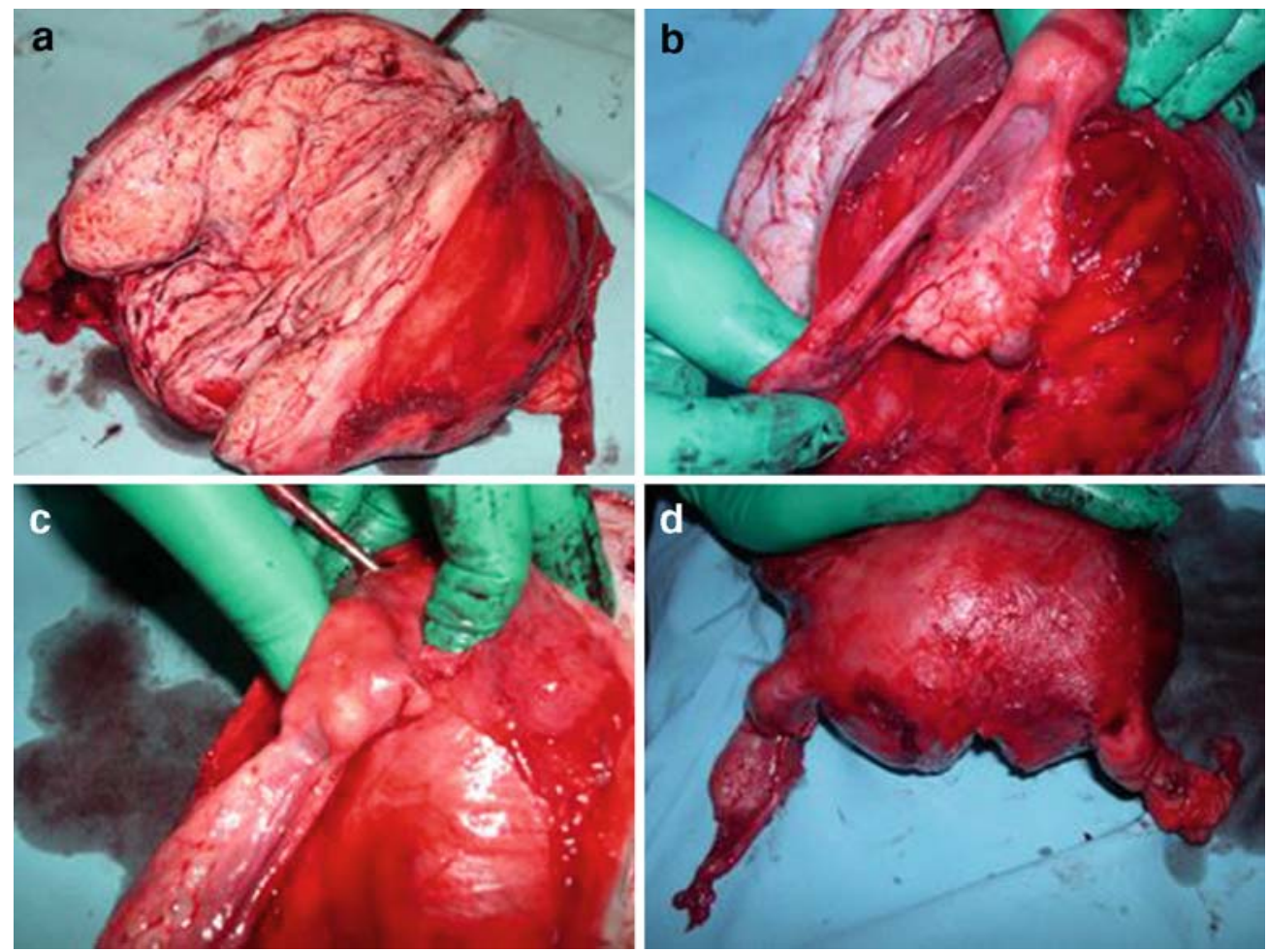

causing absent cervix and absent upper half of the vagina [1]. It is a rare Mullerian anomaly and difficult to detect [1]. Ideally, the anomaly should be completely defined before the operation. Often, however, the extent of the anomaly can only be properly determined at surgery [2]. It results from abnormal fusion of the Mullerian ducts or from failure of the septum to be absorbed; it may be associated with obstructive phenomena that lead to dysmenorrhoea and abdominopelvic masses [3]. Surgical reconstruction of the internal genitalia, restoration of menses and maintenance of a patent genital tract for these patients are challenging problems for the gynaecologist. They demonstrate the difficulty in diagnosis, heterogeneity in presentation, and need for expertise in all disorders of development of the female genital tract.

The main aim of appropriate management in this mature woman was to alleviate her physical symptom.
It is of interest that even with recent advances in gynaecology today, this lady had been seen by few doctors over the years and the fact that she has never had a period was missed and did not raise the suspicion of haematocolpos or any abnormality.

\section{References}

1. Sadler TW (1990) Langman's medical embryology, 6th edn. Williams Wilkins, Baltimore

2. Pinsonneault O, Goldstein DP (1985) Obstructing malformations of the uterus and vagina. Fertil Steril 44:241-247

3. Magee MC, Lucey DT, Fried FA (1979) A new embryonic classification for urogynecologic malformation: the syndromes of mesonephric duct induced Mullerian deformities. Urol $121: 265$ 